\title{
SOME REMARKS ON DERIVATIONS ON THE ALGEBRA OF OPERATORS IN HILBERT PRO-C*-BIMODULES
}

\author{
KH. KARIMI AND K. SHARIFI
}

\begin{abstract}
Suppose $A$ is a pro-C*-algebra. Let $L_{A}(E)$ be the pro-C*-algebra of adjointable operators on a Hilbert $A$-module $E$ and let $K_{A}(E)$ be the closed two sided *-ideal of all compact operators on $E$. We prove that if $E$ be a full Hilbert $A$-module, the innerness of derivations on $K_{A}(E)$ implies the innerness of derivations on $L_{A}(E)$. We show that if $A$ is a commutative pro- $\mathrm{C}^{*}$-algebra and $E$ is a Hilbert $A$-bimodule then every derivation on $K_{A}(E)$ is zero. Moreover, if $A$ is a commutative $\sigma$-C*-algebra and $E$ is a Hilbert $A$-bimodule then every derivation on $L_{A}(E)$ is zero, too.
\end{abstract}

\section{IntRODUCTION}

A derivation on an algebra $A$ is a linear mapping $D$ from $A$ into itself, such that $D(a b)=$ $D(a) b+a D(b)$ for all $a, b \in A$. We say that $D$ is inner if there exists $x \in A$ such that $D(a)=[a, x]=a x-x a$ for every $a \in A$. An important question in the theory of derivations is that on which algebras and under what conditions, a derivation is inner or is identically zero. In 1955, Singer and Wermer [18 proved that every derivation on a commutative Banach algebra maps the algebra into its radical, in particular, every continuous derivation on a semisimple algebra is identically zero. It is well-known that the Singer-Wermer theorem is not valid for general topological algebras [16, Corollary 2.2.4]. In 1992, Becker [2] studied derivations on a special class of topological algebras, known as pro- $\mathrm{C}^{*}$-algebras, and proved that the Singer-Wermer theorem is true for commutative pro-C*-algebras. In this paper we investigate derivations on the pro- $\mathrm{C}^{*}$-algebra $L_{A}(E)$ of all adjointable operators on a Hilbert pro-C*-bimodule $E$.

Let us quickly recall the definition of pro- $\mathrm{C}^{*}$-algebras and Hilbert modules over them. A pro-C*-algebra is a complete Hausdorff complex topological *-algebra $A$ whose topology is determined by its continuous $\mathrm{C}^{*}$-seminorms in the sense that the net $\left\{a_{i}\right\}_{i \in I}$ converges to 0 if and only if the net $\left\{p\left(a_{i}\right)\right\}_{i \in I}$ converges to 0 for every continuous $\mathrm{C}^{*}$-seminorm $p$ on $A$. For example the algebra $C(X)$ of all continuous complex valued functions on a

2010 Mathematics Subject Classification. Primary 46L08; Secondary 46L05, 46H25, $47 \mathrm{~B} 47$.

Key words and phrases. Hilbert pro-C*-bimodule, pro-C*-algebra, derivation, compact operator. 
compactly generated space or a CW complex $X$, the cartesian product $\prod_{\alpha \in I} A_{\alpha}$ of $\mathrm{C}^{*}$ algebras $A_{\alpha}$ with the product topology are pro-C*-algebras [4, §7.6]. A $\sigma-C^{*}$-algebra is a pro- $\mathrm{C}^{*}$-algebra whose topology is determined by a countable family of $\mathrm{C}^{*}$-seminorms. Pro$\mathrm{C}^{*}$-algebras appear in the study of certain aspects of $\mathrm{C}^{*}$-algebras such as tangent algebras of $\mathrm{C}^{*}$-algebras, domain of closed *-derivations on $\mathrm{C}^{*}$-algebras, multipliers of Pedersen's ideal, and noncommutative analogues of classical Lie groups. These algebras were first introduced by Inoue [6] who called them locally $\mathrm{C}^{*}$-algebras and studied more in [1, 4, 12] with different names. A right pre-Hilbert module over a pro- $\mathrm{C}^{*}$-algebra $A$ is a right $A$-module $E$, compatible with the complex algebra structure, equipped with an $A$-valued inner product $\langle\cdot, \cdot\rangle_{A}: E \times E \rightarrow A(x, y) \mapsto\langle x, y\rangle_{A}$, which is $A$-linear in the second variable $y$ and has the properties:

$$
\langle x, y\rangle_{A}=\langle y, x\rangle_{A}^{*} \text {, and }\langle x, x\rangle_{A} \geq 0 \text { with equality if and only if } x=0 .
$$

A right pre-Hilbert $A$-module $E$ is a right Hilbert $A$-module if $E$ is complete with respect to the topology determined by the family of seminorms $\left\{\bar{p}^{A}\right\}_{p \in S(A)}$ where $\bar{p}^{A}(\xi)=\sqrt{p\left(\langle\xi, \xi\rangle_{A}\right)}$, $\xi \in E$. The notion of a left Hilbert $A$-module is defined in an analogous way. Denote by $\langle E, E\rangle_{A}$ the closure of the linear span of all $\langle x, y\rangle_{A}, x, y \in E$. We call $E$ is full if $\langle E, E\rangle_{A}=A$. One can always consider any right Hilbert module over pro- $\mathrm{C}^{*}$-algebra $A$ as a full Hilbert module over pro-C*-algebra $\langle E, E\rangle_{A}$. Hilbert modules over pro-C*-algebras have been studied in the book [7] and the papers [8, 12, 17].

In 1992, Becker [2, Proposition 2] proved that every derivation on a pro-C*-algebra is continuous. He also proved that commutative pro- $\mathrm{C}^{*}$-algebras have no nonzero derivations [2, Corollary 3]. In 1995, Phillips [13, Theorem 3] proved that every derivation on a pro$\mathrm{C}^{*}$-algebra is approximately inner. A result of J. Ringrose states that every derivation of a $\mathrm{C}^{*}$-algebra $A$ into a Banach $A$-bimodule is continuous. Weigt and Zarakas [19] proved some extensions of this result in the context of pro- $\mathrm{C}^{*}$-bimodules and complete locally convex bimodules. In [20], Zarakas introduced the notion of a Hilbert pro- $\mathrm{C}^{*}$-bimodule and proved that every derivation of a pro- $\mathrm{C}^{*}$-algebra into a Hilbert pro- $\mathrm{C}^{*}$-bimodule is continuous. For a detailed survey of derivations of pro- $\mathrm{C}^{*}$-algebras, see [5].

Let $A$ be a pro- $C^{*}$-algebra and $E$ be a right Hilbert module over $A$. A map $T: E \rightarrow E$ is called adjointable if there exists a map $T^{*}: E \rightarrow E$ with the property $\langle T x, y\rangle_{A}=\left\langle x, T^{*} y\right\rangle_{A}$ for all $x, y \in E$. The set $L_{A}(E)$ of all adjointable maps on $E$ is a pro- $\mathrm{C}^{*}$-algebra with the topology defined by the family of $\mathrm{C}^{*}$-seminorms $\{\tilde{p}\}_{p \in S(A)}$, in which, $\tilde{p}(T)=\sup \left\{\bar{p}^{A}(T x)\right.$ : $\left.x \in E, \bar{p}^{A}(x) \leq 1\right\}$. The set of all compact operators $K_{A}(E)$ on $E$ is defined as the closure of 
the set of all finite linear combinations of the operators $\left\{\theta_{x, y}: \theta_{x, y}(\xi)=x\langle y, \xi\rangle_{A}, x, y, \xi \in E\right\}$. It is a pro- ${ }^{*}$-subalgebra and a two sided ideal of $L_{A}(E)$.

Derivations on the algebra of operators on Hilbert $C^{*}$-modules have been recently studied in the papers [11, 14, 15]. Li et al. [10, Theorem 2.3] studied the relation between the innerness of derivations on $K_{A}(E)$ and $L_{A}(E)$ and proved that if $A$ is a $\sigma$-unital commutative $\mathrm{C}^{*}$-algebra and $E$ is a full Hilbert $A$-module, then every derivation on $L_{A}(E)$ is inner if every derivation on $K_{A}(E)$ is inner. In this paper, we prove the above result in the context of pro$\mathrm{C}^{*}$-algebras and we show that the assumptions of $\sigma$-unitality and commutativity of $A$ are not required in this theorem. We also consider derivations on the algebra of operators of Hilbert pro- $\mathrm{C}^{*}$-bimodules and we prove that if $A$ is a commutative pro- $\mathrm{C}^{*}$-algebra and $E$ is a Hilbert $A$-bimodule, then every derivation on $K_{A}(E)$ is zero. Furthermore, we show that if $A$ is a commutative $\sigma$ - $C^{*}$-algebra and $E$ is a Hilbert $A$-bimodule, then every derivation on $L_{A}(E)$ is zero.

\section{Preliminaries}

Let $A$ be a pro- $\mathrm{C}^{*}$-algebra and let $S(A)$ be the set of all continuous $\mathrm{C}^{*}$-seminorms on $A$ and $p \in S(A)$. We set $N_{p}=\{a \in A: p(a)=0\}$ then $A_{p}=A / N_{p}$ is a $\mathrm{C}^{*}$-algebra in the norm induced by $p$. For $p, q \in S(A)$ with $p \geq q$, the surjective morphisms $\pi_{p q}: A_{p} \rightarrow A_{q}$ defined by $\pi_{p q}\left(a+N_{p}\right)=a+N_{q}$ induce the inverse system $\left\{A_{p} ; \pi_{p q}\right\}_{p, q \in S(A), p \geq q}$ of $\mathrm{C}^{*}$-algebras and $A=\lim _{\longleftarrow} A_{p}$, i.e. the pro- $\mathrm{C}^{*}$-algebra $A$ can be identified with $\lim _{p} A_{p}$. The canonical map from $A$ onto $A_{p}$ is denoted by $\pi_{p}$ and $a_{p}$ is reserved to denote $a+N_{p}$. A morphism of pro- $\mathrm{C}^{*}$-algebras is a continuous morphism of $*$-algebras. An isomorphism of pro- $\mathrm{C}^{*}$-algebras is a morphism of pro- $\mathrm{C}^{*}$-algebras which possesses an inverse morphism of pro- $\mathrm{C}^{*}$-algebras.

An approximate unit of a pro- $C^{*}$-algebra $A$ is an increasing net $\left\{e_{i}\right\}_{i \in I}$ of positive elements of $A$ such that $p\left(e_{i}\right) \leq 1$ for all $i \in I$ and $p \in S(A) ; p\left(a e_{i}-a\right) \rightarrow 0$ and $p\left(e_{i} a-a\right) \rightarrow 0$ for all $p \in S(A)$ and $a \in A$. Any pro- $\mathrm{C}^{*}$-algebra has an approximate unit.

Let $E$ be a left and right Hilbert module over a pro-C*-algebra $A$. Denote by ${ }_{A}\langle\cdot, \cdot\rangle$ and $\langle\cdot, \cdot\rangle_{A}$ the left and right $A$-valued inner products on $E$. Then there are two locally convex topologies defined on $E$. One denoted by $\tau^{A}$, induced by the seminorms $\left\{\bar{p}^{A}\right\}_{p \in S(A)}$ corresponding to its structure as a right Hilbert $A$-module and the other, denoted by ${ }^{A} \tau$, induced by the seminorms $\left\{{ }^{A} \bar{p}\right\}_{p \in S(A)}$, corresponding to its structure as a left Hilbert $A$ module. Zarakas [20, Corollary 3.2] proved that if ${ }_{A}\langle x, y\rangle z=x\langle y, z\rangle_{A}$ for all $x, y, z \in E$ and we assume continuity of the left (resp. right) module action, in the sense that

$$
\bar{p}^{A}(a x) \leq p(a) \bar{p}^{A}(x), \quad{ }^{A} \bar{p}(x a) \leq{ }^{A} \bar{p}(x) p(a), \quad x \in E, a \in A,
$$


then $\bar{p}^{A}(x)={ }^{A} \bar{p}(x)$, for all $x \in E$ and so two topologies ${ }^{A} \tau$ and $\tau^{A}$ on $E$ coincide. Based on this fact he defined the notion of Hilbert pro- $\mathrm{C}^{*}$-bimodule as follows.

Definition 2.1. Let $A$ be a pro- $C^{*}$-algebra and $E$ be a left and right Hilbert $A$-module. If condition (2.1) is satisfied and ${ }_{A}\langle x, y\rangle z=x\langle y, z\rangle_{A}$ for all $x, y, z \in E$, then we say that $E$ is a Hilbert pro- $C^{*}$-bimodule over $A$ or a Hilbert $A$-bimodule.

Suppose $E$ is a right Hilbert $A$-module. For each $p \in S(A), N_{p}^{E}=\left\{\xi \in E: \bar{p}^{A}(\xi)=0\right\}$ is a closed submodule of $E$ and $E_{p}=E / N_{p}^{E}$ is a right Hilbert $A_{p}$-module with the action $\left(\xi+N_{p}^{E}\right) \pi_{p}(a)=\xi a+N_{p}^{E}$ and the inner product $\left\langle\xi+N_{p}^{E}, \eta+N_{p}^{E}\right\rangle_{A_{p}}=\pi_{p}\left(\langle\xi, \eta\rangle_{A}\right)$. The canonical map from $E$ onto $E_{p}$ is denoted by $\sigma_{p}^{E}$ and $\xi_{p}$ is reserved to denote $\sigma_{p}^{E}(\xi)$. For $p, q \in$ $S(A)$ with $p \geq q$, the surjective morphisms $\sigma_{p q}^{E}: E_{p} \rightarrow E_{q}$ defined by $\sigma_{p q}^{E}\left(\sigma_{p}^{E}(\xi)\right)=\sigma_{q}^{E}(\xi)$ induce the inverse system $\left\{E_{p} ; A_{p} ; \sigma_{p q}^{E}, \pi_{p q}\right\}_{p, q \in S(A), p \geq q}$ of right Hilbert $\mathrm{C}^{*}$-modules. In this case, $\lim _{\llcorner} E_{p}$ is a right Hilbert $A$-module which can be identified with $E$. Let $E$ and $F$ be right Hilbert $A$-modules and $T: E \rightarrow F$ be an $A$-module map. The module map $T$ is called bounded if for each $p \in S(A)$, there is $k_{p}>0$ such that $\bar{p}_{F}^{A}(T x) \leq k_{p} \bar{p}_{E}^{A}(x)$ for all $x \in E$. The set $L_{A}(E, F)$ of all bounded adjointable $A$-module maps from $E$ into $F$ becomes a locally convex space with the topology defined by the family of seminorms $\{\tilde{p}\}_{p \in S(A)}$, where $\tilde{p}(T)=$ $\left\|\left(\pi_{p}\right)_{*}(T)\right\|_{L_{A_{p}}\left(E_{p}, F_{p}\right)}$ and $\left(\pi_{p}\right)_{*}: L_{A}(E, F) \rightarrow L_{A_{p}}\left(E_{p}, F_{p}\right)$ is defined by $\left(\pi_{p}\right)_{*}(T)\left(\xi+N_{p}^{E}\right)=$ $T \xi+N_{p}^{F}$, for all $T \in L_{A}(E, F)$ and $\xi \in E$. For $p, q \in S(A)$ with $p \geq q$, the morphisms $\left(\pi_{p q}\right)_{*}$ : $L_{A_{p}}\left(E_{p}, F_{p}\right) \rightarrow L_{A_{q}}\left(E_{q}, F_{q}\right)$ defined by $\left(\pi_{p q}\right)_{*}\left(T_{p}\right)\left(\sigma_{q}^{E}(\xi)\right)=\sigma_{p q}^{F}\left(T_{p}\left(\sigma_{p}^{E}(\xi)\right)\right)$ induce the inverse system $\left\{L_{A_{p}}\left(E_{p}, F_{p}\right) ;\left(\pi_{p q}\right)_{*}\right\}_{p, q \in S(A), p \geq q}$ of Banach spaces such that $\lim _{p} L_{A_{p}}\left(E_{p}, F_{p}\right)$ can be identified to $L_{A}(E, F)$. In particular, topologizing, $L_{A}(E, E)$ becomes a pro-C*-algebra which is abbreviated by $L_{A}(E)$. The set of all compact operators $K_{A}(E)$ on $E$ is defined as the closed linear subspace of $L_{A}(E)$ spanned by $\left\{\theta_{x, y}: \theta_{x, y}(\xi)=x\langle y, \xi\rangle\right.$ for all $x, y, \xi \in$ $E\}$. This is a pro- $C^{*}$-subalgebra and a two sided ideal of $L_{A}(E)$, moreover, $K_{A}(E)$ can be identified to $\lim _{\longleftarrow} K_{A_{p}}\left(E_{p}\right)$.

\section{MAin RESUlts}

The following theorem states that the innerness of derivations on $K_{A}(E)$ implies the innerness of derivations on $L_{A}(E)$. By this theorem, the assumption of $\sigma$-unitality and commutativity of $A$ in [10, Theorem 2.3] can be removed.

Theorem 3.1. Let $A$ be a pro- $C^{*}$-algebra and let $E$ be a full right Hilbert $A$-module. If every derivation on $K_{A}(E)$ is inner, then any derivation on $L_{A}(E)$ is also inner. 
Proof. Let $D$ be a derivation on $L_{A}(E)$. We claim that $D$ maps $K_{A}(E)$ to itself. To see this, suppose $x, y \in E$ and apply [7, Corollary 1.3.11], there exist $a \in A$ and $z \in E$ such that $x=z a$. Since $E$ is full, there exists a sequence $\left\{a_{n}\right\}$ in $\langle E, E\rangle$ such that $p\left(a_{n}-a\right) \rightarrow 0$ for all $p \in S(A)$. Let $a_{n}=\sum_{i=1}^{k_{n}}\left\langle x_{i n}, y_{i n}\right\rangle$ where $x_{i n}, y_{i n} \in E$. We have

$$
\begin{aligned}
D\left(\theta_{z a_{n}, y}\right)=\sum_{i=1}^{k_{n}} D\left(\theta_{z\left\langle x_{i n}, y_{i n}\right\rangle, y}\right) & =\sum_{i=1}^{k_{n}} D\left(\theta_{z, x_{i n}} \theta_{y_{i n}, y}\right) \\
& =\sum_{i=1}^{k_{n}} D\left(\theta_{z, x_{i n}}\right) \theta_{y_{i n}, y}+\sum_{i=1}^{k_{n}} \theta_{z, x_{i n}} D\left(\theta_{y_{i n}, y}\right) \in K_{A}(E) .
\end{aligned}
$$

Let $p \in S(A)$ and $w \in E$ such that $\bar{p}^{A}(w) \leq 1$ then

$$
\begin{aligned}
\bar{p}^{A}\left(\left(\theta_{z a_{n}, y}-\theta_{z a, y}\right)(w)\right)^{2} & =p\left(\langle y, w\rangle^{*}\right) p\left(\left\langle z a_{n}-z a, z a_{n}-z a\right\rangle\right) p(\langle y, w\rangle) \\
& \leq \bar{p}^{A}(y)^{2} \bar{p}^{A}(z)^{2} p\left(a_{n}-a\right)^{2}
\end{aligned}
$$

which implies that $\tilde{p}\left(\theta_{z a_{n}, y}-\theta_{x, y}\right) \rightarrow 0$. Consequently, $\theta_{z a_{n}, y} \rightarrow \theta_{x, y}$ in $L_{A}(E)$ with respect to the topology generated by $\{\tilde{p}\}_{p \in S(A)}$. It follows from [2, Proposition 2] that $D$ maps $K_{A}(E)$ into itself. Since every derivation on $K_{A}(E)$ is inner, there exists $T \in K_{A}(E)$ such that $D(K)=T K-K T$, for all $K \in K_{A}(E)$. Let $S \in L_{A}(E)$ and $x, y \in E$ then $D\left(S \theta_{x, y}\right)=$ $T S \theta_{x, y}-S \theta_{x, y} T$. The latter equality and the fact that

$$
D\left(S \theta_{x, y}\right)=D(S) \theta_{x, y}+S D\left(\theta_{x, y}\right)=D(S) \theta_{x, y}+S T \theta_{x, y}-S \theta_{x, y} T,
$$

imply $D(S) \theta_{x, y}=T S \theta_{x, y}-S T \theta_{x, y}$. Let $w \in E$ then there exist $z \in E$ and $a \in A$ such that $w=z a$. Since $E$ is full, there exists a sequence $\left\{a_{n}\right\}$ in $\langle E, E\rangle$ such that $p\left(a_{n}-a\right) \rightarrow 0$ for all $p \in S(A)$. Let $a_{n}=\sum_{i=1}^{k_{n}}\left\langle x_{i n}, y_{i n}\right\rangle$ then

$$
\begin{aligned}
D(S)\left(z a_{n}\right)=\sum_{i=1}^{k_{n}} D(S)\left(z\left\langle x_{i n}, y_{i n}\right\rangle\right) & =\sum_{i=1}^{k_{n}} D(S) \theta_{z, x_{i n}}\left(y_{i n}\right) \\
& =\sum_{i=1}^{k_{n}}\left(T S \theta_{z, x_{i n}}-S T \theta_{z, x_{i n}}\right)\left(y_{i n}\right) \\
& =(T S-S T)\left(z a_{n}\right) .
\end{aligned}
$$

For all $p \in S(A), \bar{p}_{A}\left(z a_{n}-z a\right) \leq \bar{p}_{A}(z) p\left(a_{n}-a\right)$ and so $z a_{n} \rightarrow z a$ in $E$. Hence, continuity of $D(S)$ and $T S-S T$ implies that $D(S)(w)=(T S-S T)(w)$ for all $w \in E$, that is, $D(S)=T S-S T$.

Remark 3.2. Let $A$ be a $\sigma$-C $\mathrm{C}^{*}$-algebra and let $E$ be a Hilbert $A$-bimodule. Denote by ${ }_{A} I$ the closure of the two-sided ideal $\operatorname{span}\left\{{ }_{A}\langle x, y\rangle: x, y \in E\right\}$. By [20, Lemma 6.4], ${ }_{A} I$ has an 
approximate unit $\left\{u_{\alpha}\right\}$ with $u_{\alpha}=\sum_{i=1}^{n} A_{A}\left\langle x_{i}^{\alpha}, x_{i}^{\alpha}\right\rangle$, where $\alpha=\left\{y_{1}, \ldots, y_{n}\right\} \subset E$ ranges over finite subsets of $E$ and $x_{i}^{\alpha}=\left(\sum_{j=1}^{n} A\left\langle y_{j}, y_{j}\right\rangle+\frac{1}{n} 1\right)^{-\frac{1}{2}} y_{i}, i=1, \ldots, n$, in which 1 is the identity of the unitization of $A$. Zarakas considered a realization of $K_{A}(E)$, through the closed twosided $*$-ideal ${ }_{A} I$. Indeed, he proved that ${ }_{A} I \simeq K_{A}(E)$ is a topological $*$-isomorphism [20, Theorem 6.5]. This theorem can be regarded as an extension of a result of Brown, Mingo and Shen [3, proposition 1.10] for Hilbert $C^{*}$-bimodules. In view of this fact, it is easy to see that $\left\{T_{\alpha}\right\}$, where $T_{\alpha}=\sum_{i=1}^{n} \theta_{x_{i}^{\alpha}, x_{i}^{\alpha}}$, is an approximate unit for $K_{A}(E)$.

Lemma 3.3. Every derivation of a pro- $C^{*}$-algebra annihilates its center.

Proof. Let $D$ be a derivation on a pro-C*-algebra $A$. For each $p \in S(A)$, we consider the linear map $D_{p}: A_{p} \rightarrow A_{p}$ defined by $D_{p}\left(\pi_{p}(a)\right)=\pi_{p}(D(a))$, where $a \in A$. Let $a \in N_{p}$. By [2, Lemma 1], there exist elements $a_{k} \in N_{p}, k=1, \ldots, 4$ such that $a=\sum_{k=1}^{4} i^{k} a_{k}^{2}$. Since $p(D(a)) \leq \sum_{k=1}^{4} p\left(D\left(a_{k}\right) a_{k}+a_{k} D\left(a_{k}\right)\right)=0$, we have $D(a) \in N_{p}$ which shows that $D_{p}$ is well-defined. It is easy to see that $D_{p}$ is a derivation on $A_{p}$. Denote by $Z(A)$ the center of $A$ and let $a \in Z(A)$. Then $\pi_{p}(a)$ is in the center of $A_{p}$, for every $p \in S(A)$. It is well-known that every derivation on a $\mathrm{C}^{*}$-algebra annihilates its center [9, Theorem 2], so $D_{p}\left(\pi_{p}(a)\right)=0$ for every $p \in S(A)$. It follows that $\pi_{p}(D(a))=0$ for all $p \in S(A)$. Since $S(A)$ is a separating family of $\mathrm{C}^{*}$-seminorms, we have $D(a)=0$.

Remark 3.4. Let $A$ be a pro- $C^{*}$-algebra and let $E$ be a Hilbert $A$-bimodule. If $A$ is commutative, then $K_{A}(E)$ is commutative. Indeed, for every $x, y, z, u, v \in E$ we have

$$
\begin{aligned}
\theta_{x, y} \theta_{u, v}(z)=\theta_{x \cdot\langle y, u\rangle_{A}, v}(z)=x \cdot\langle y, u\rangle_{A}\langle v, z\rangle_{A} & ={ }_{A}\langle x, y\rangle u \cdot\langle v, z\rangle_{A} \\
& ={ }_{A}\langle x, y\rangle_{A}\langle u, v\rangle . z
\end{aligned}
$$

and

$$
\begin{aligned}
\theta_{u, v} \theta_{x, y}(z)=\theta_{u .\langle v, x\rangle_{A}, y}(z)=u \cdot\langle v, x\rangle_{A}\langle y, z\rangle_{A} & ={ }_{A}\langle u, v\rangle x \cdot\langle y, z\rangle_{A} \\
& ={ }_{A}\langle u, v\rangle_{A}\langle x, y\rangle . z .
\end{aligned}
$$

Therefore $\theta_{x, y} \theta_{u, v}=\theta_{u, v} \theta_{x, y}$ for all $x, y \in E$, which implies the commutativity of $K_{A}(E)$.

Corollary 3.5. Let $A$ be a pro- $C^{*}$-algebra and let $E$ be a Hilbert $A$-bimodule. If $A$ is commutative then every derivation on $K_{A}(E)$ is zero.

The proof follows from Proposition 3.4, Lemma 3.3 and the fact that $K_{A}(E)$ is a pro-C*algebra. 
Theorem 3.6. Let $A$ be a commutative $\sigma-C^{*}$-algebra and let $E$ be a Hilbert A-bimodule. Then every derivation on $L_{A}(E)$ is zero.

Proof. Let $D$ be a derivation on $L_{A}(E)$ and let $\left\{T_{\alpha}\right\}$ be as in Remark 3.2. First we show that $D$ maps $K_{A}(E)$ to itself. Let $x, y \in E$ then $D\left(\theta_{x, y} T_{\alpha}\right)=\sum_{i=1}^{n} D\left(\theta_{x, y} \theta_{x_{i}^{\alpha}, x_{i}^{\alpha}}\right)=$ $\sum_{i=1}^{n} D\left(\theta_{x, y}\right) \theta_{x_{i}^{\alpha}, x_{i}^{\alpha}}+\sum_{i=1}^{n} \theta_{x, y} D\left(\theta_{x_{i}^{\alpha}, x_{i}^{\alpha}}\right) \in K_{A}(E)$. Continuity of $D$ and closeness of $K_{A}(E)$ in $L_{A}(E)$ imply that $D\left(\theta_{x, y}\right) \in K_{A}(E)$ for all $x, y \in E$. Consequently, the restriction of $D$ to $K_{A}(E)$ is a derivation on $K_{A}(E)$ which is zero by Corollary 3.5. Let $S \in L_{A}(E)$ then $D(S) \theta_{x, y}=D\left(S \theta_{x, y}\right)-S D\left(\theta_{x, y}\right)=0$ for all $x, y \in E$. In particular, $D(S) \theta_{x, D(S) x}=0$ and so

$$
\begin{aligned}
D(S)(x)\langle D(S)(x), D(S)(x)\rangle & =D(S)(x\langle D(S)(x), D(S)(x)\rangle) \\
& =D(S) \theta_{x, D(S)(x)}(D(S)(x)) \\
& =0 .
\end{aligned}
$$

Therefore, $|D(S)(x)|^{4}=\langle D(S)(x)\langle D(S)(x), D(S)(x)\rangle, D(S)(x)\rangle=0$, i.e. $D(S)(x)=0$, for all $x \in E$.

Acknowledgement: The authors would like to thank the referee for his/her careful reading and useful comments.

\section{REFERENCES}

[1] C. Apostol, b*-algebras and their representaion, J. London Math. Soc. 3 (1971), 30-38.

[2] R. Becker, Derivations on LMC*-algebras, Math. Nachr. 155 (1992), 141-149.

[3] L. G. Brown, J. A. Mingo and N. T. Shen, Quasimultipliers and embeddings of Hilbert $\mathrm{C}^{*}$-modules. Canad. J. Math. 46 (1994), 1150-1174.

[4] M. Fragoulopoulou, Topological algebras with involution, North Holland, Amsterdam, 2005.

[5] M. Fragoulopoulou, M. Weigt and I. Zarakas, Derivations of locally convex *-algebras, Extracta Math. 26 (2011), 45-60.

[6] A. Inoue, Locally C*-algebras, Mem. Faculty Sci. Kyushu Univ. Ser. A 25 (1971), 197-235.

[7] M. Joita, Hilbert modules over locally $C^{*}$-algebras, University of Bucharest Press, 2006.

[8] M. Joita, Crossed products of pro-C*-algebras and Hilbert pro-C*-modules, Bull. Malays. Math. Sci. Soc. 38 (2015), 1053-1065

[9] R. V. Kadison, Derivations of operator algebras, Ann. Math., 83(2) (1966), 280-293.

[10] P. Li, D. Han and W. Tang, Derivations on the algebra of operatores in Hilbert C*-modules, Acta Math. Sinica, English ser. 28 (8) (2012), 1615-1622.

[11] M. K. Moghadam, M. Miri and A. R. Janfada, A note on derivations on the algebra of operators in Hilbert C*-modules, Mediterr. J. Math. DOI 10.1007/s00009-015-0538-y, Published online 25 February 2015.

[12] N. C. Phillips, Inverse limit of C*-algebras, J. Operator Theory 19 (1988), 159-195. 
[13] N. C. Phillips, Inner derivations on $\sigma$-C*-algebras, Math. Nachr. 176, (1995), 243-247.

[14] A. Sahleh and L. Najarpisheh, Derivations of operators on Hilbert modules, Gen. Math. Notes 24 (2014), $52-57$.

[15] H. Saidi, A. R. Janfada and M Mirzavaziri, Kinds of derivations on Hilbert $\mathrm{C}^{*}$-modules and their operator algebras, Miskolc Mathematical Notes 16 (2015), 453-461.

[16] S. Sakai, Operator algebras in dynamical systems: The theory of unbounded derivations in $C^{*}$-algebras, Encyclipedia of Mathematics and its applications, 41, Cambridge University Press, 1991.

[17] K. Sharifi, Generic properties of module maps and characterizing inverse limits of $\mathrm{C}^{*}$-algebras of compact operators, Bull. Malays. Math. Sci. Soc. 36 (2013), 481-489.

[18] I. M. Singer and J. Wermer, Derivations on commutative normed algebras, Math. Ann. 126 (1955), 260-264.

[19] M. Weigt and I. Zarakas, A note on derivations of pro-C*-algebras into complete locally convex bimodules, New Zealand J. Math 41 (2011), 143-152.

[20] I. Zarakas, Hilbert pro-C*-bimodules and applications, Rev. Roumaine Math. Pures Appl. 17 (2012), 289-310.

KHADIJEH KARIMI,

Department of Mathematics, Shahrood University of Technology, P. O. Box 3619995161 316, SHAHROOD, IRAN.

E-mail address: kh_karimi5005@yahoo.com

KAMRAN SHARIFI,

Department of Mathematics, Shahrood University of Technology, P. O. Box 3619995161 316, Shahrood, Iran.

E-mail address: sharifi.kamran@gmail.com 